\title{
INFLUENCE OF CONFIGURATION INTERACTION ON THE MAGNETIC CIRCULAR DICHROISM SPECTRA OF $\mathrm{C}_{60}$.MOLECULE
}

\author{
M. Pilch and M. Pawlikowski \\ Department of Theoretical Chemistry, Jagiellonian University \\ Ingardena 3, 30-060 Kraków, Poland
}

(Received July 30, 1993)

\begin{abstract}
$A_{1} / D_{0}$ and $D_{0}$ characteristics of magnetic circular dichroism and absorption spectra are studied for the low-energy $A_{\mathrm{g}} \rightarrow T_{1 \mathrm{u}}$ transitions in the $\mathrm{C}_{60}$ molecule in terms of the self-consistent field Parieser-Parr-Pople method applied in the full configuration interaction-1 treatment. The effects are discussed at different levels of configuration interaction and the results are compared to the earlier ones obtained from the CNDO/S method. We argue that the earlier treatment fails to accc unt correctly for the experimental magnetic circular dichroism and absorption spectra of $\mathrm{C}_{60}$ molecule. This is most probably due to deficiency of the ccnfiguration interaction basis set and/or to the intrinsic parametrization of complete neglect of differential overlap method.
\end{abstract}

PACS numbers: 33.55.Be

\section{Introduction}

In the recent paper [1] we have discussed the magneto-optical properties of $\mathrm{C}_{60}$ molecule in terms of self-consistent field Parieser-Parr-Pople (SCFPPP) method suitably adapted to study molecules with three-dimensional $\pi$-systems. Using such an adapted method we showed that the experimental magnetic circular dichroism (MCD) and absorption spectra corresponding to the lowest energy $A_{\mathrm{g}} \rightarrow T_{1 \mathrm{u}}$ transitions can be reasonably well :eproduced in an approximate configuration interaction (CI) treatment which encompasses all singly excited configurations due to $\pi \rightarrow \pi^{*}$ single promotions ir. the $\pi$ system of $\mathrm{C}_{60}$ molecule. A comparison between our results [1] and those obtained with the quantum chemical force field/ $n$ electrons (QCFF/PI) method [2] was moderately successful because of minor discrepancies in the energies and the intensities of the QCFF/PI and SCFPPP parametrizations. Most recently, some magneto-optical properties of $\mathrm{C}_{60}$ molecule were also studied with complete neglect of differential overlap (CNDO/S) 
method applied with the limited number of 171 and 196 configurations. At this level of CI approach, Marconi and Salvi [3] have concluded that: "for the lowest permitted states of $T_{1 \mathrm{u}}$ symmetry the $A$ term is well reproduced in size and sign independently from the parametrization and the amount of configuration interaction used". We do not agree with that conclusion as the CNDO/S method used with the limited number of configurations yields the intensities and the magnetic moments of the $T_{1 \mathrm{u}}$-symmetry states to be strongly at variance with the MCD experiment [4] and also with our later calculations [1]. Moreover, the energies and intensities calculated for the three low-energy $T_{1 \mathrm{u}}$ states obtained by Marconi and Salvi $[3,5]$ are also inconsistent with those recently found using the CNDO/S method employed at the same level of CI treatment [6]. Using SCFPPP method as a guide, the possible causes of these inconsistencies are discussed in certain detail in this paper.

\section{Results and discussion}

For purpose of this paper we will compare the energies, $E$, the absorption coefficients, $D_{0}$, and the MCD ratios, $A_{1} / D_{0}$, calculated for the low-energy $A_{\mathrm{g}} \rightarrow T_{1 \mathrm{u}}$ transitions using CNDO/S and SCFPPP method applied at different levels of CI. The results required in our discussion are collected in Table. The CI

TABLE

Comparison between SCFPPP (A) and CNDO/S (B,C and D) calculations for the lowest energy singlet states of fullerene: (A) the result of this paper; (B) and (C) Ref. [3]; (D) Ref. [6].

\begin{tabular}{c|c|c|c|c|c|c|c|c|c|c|c}
\hline \hline & \multicolumn{3}{|c|}{$\mathrm{CI}=900(\mathrm{~A})$} & \multicolumn{3}{c|}{$\mathrm{CI}=171(\mathrm{~B})$} & \multicolumn{3}{c|}{$\mathrm{CI}=196(\mathrm{C})$} & \multicolumn{2}{c}{$\mathrm{CI}=196(\mathrm{D})$} \\
\cline { 2 - 12 } & $E^{a}$ & $A_{1} / D_{0}^{b}$ & $D_{0}^{c}$ & $E^{a}$ & $A_{1} / D_{0}^{b}$ & $D_{0}^{c}$ & $E^{a}$ & $A_{1} / D_{0}^{b}$ & $D_{0}^{c}$ & $E^{a}$ & $D_{0}^{c}$ \\
\hline $1 T_{1 \mathrm{u}}$ & 3.79 & -2.05 & 1.00 & 3.86 & -0.65 & 1.00 & 3.57 & -1.00 & 1.00 & 3.49 & 1.00 \\
$2 T_{1 \mathrm{u}}$ & 4.20 & -1.50 & 0.08 & 4.61 & -0.88 & 4.18 & 4.15 & -0.40 & 1.55 & 4.08 & 0.71 \\
$3 T_{1 \mathrm{u}}$ & 4.91 & 0.25 & 4.55 & 5.99 & -0.82 & 3.75 & 5.19 & -0.21 & 184.0 & 5.02 & 43.10 \\
$4 T_{1 \mathrm{u}}$ & 5.58 & 1.24 & 3.37 & - & - & - & - & - & - & 5.34 & 17.14 \\
$5 T_{1 \mathrm{u}}$ & 6.16 & -0.25 & 0.34 & - & - & - & - & - & - & 5.84 & 67.62 \\
\hline
\end{tabular}

a In eV units.

b In Bohr magneton units.

${ }^{c}$ Relative units.

$A_{1} / D_{0}$ not given in Ref. [6].

treatment leading to the numbers in the part $\mathrm{A}$ of Table comprises 900 configurations due to single electron promotion from the all filled shells to the all empty ones of the $\pi$-system [1]. The results in the parts B and C and D are those reported in Refs. [3] and [6]. They correspond to the following schemes of single electron promotions:

\section{Scheme B}

$$
1 g_{\mathrm{g}}, 1 h_{\mathrm{u}} \rightarrow 2 \mathrm{t}_{1 \mathrm{u}}, 1 \mathrm{t}_{1 \mathrm{~g}}, 3 h_{\mathrm{g}}, 2 \mathrm{t}_{1 \mathrm{u}}, 2 h_{\mathrm{u}}
$$


Scheme C and D

$1 g_{\mathrm{g}}, 1 h_{\mathrm{g}}, 1 h_{\mathrm{u}} \rightarrow 2 \mathrm{t}_{1 \mathrm{u}}, 1 \mathrm{t}_{1 \mathrm{~g}}, 3 h_{\mathrm{g}}, 2 \mathrm{t}_{2 \mathrm{u}}$.

The symmetry arguments applicable to the molecules belonging to $I_{h}$ point group show that the schemes $\mathrm{B}$ and $\mathrm{C}$ offer, respectively, 4 and 5 configurations of $T_{1 \mathrm{u}}$-symmetry. With a priori the same CNDO/S parametrization [3] applied to reach the numbers in column $\mathrm{B}$ and $\mathrm{C}$ of Table, one can expect that the increase in the number of functions taken in CI treatment showed a result in the decrease in energies of $T_{1 \mathrm{u}}$-symmetry states.

In fact, such a trend is seen in Table. However, the CNDO/S method used with 171 configurations fits the experimental energies [4] at $E\left(1 T_{1 \mathrm{u}}\right)=3.80 \mathrm{eV}$, $E\left(2 T_{1 \mathrm{u}}\right)=4.90 \mathrm{eV}, E\left(3 T_{1 \mathrm{u}}\right)=5.96 \mathrm{eV}$, much better than CNDO/S method does with the larger number of 196 configurations. Specifically, the CNDO/S calculations with 196 configurations lead to energies which are significantly lower than those found in the experiment. Therefore, it is conceivable that even more configurations required in principle to improve the correlation energy will tend to lower the transition energies beyond the limits imposed by the experiment. To reverse this trend in such an extended CI treatment, the inherent CNDO/S parametrization must be properly adjusted. It can, and generally will, lead to results which differ from those given in part B and C of Table. Therefore, in contrast with the conclusion of Refs. [3] and [5] the results of CNDO/S calculations depend not only on the parametrization but also on the number of configuration used in the actual calculations. On the other hand, comparing the intensities and the energies in part $\mathrm{C}$ and D of Table, we face something puzzling. Both $\mathrm{C}$ and D sets of number were obtained from CNDO/S method employed with the same number (196) of configurations and with the same (Mataga-Nishimoto) parametrization for the repulsion integrals. As the details of the CNDO/S parametrization were not given in Refs. $[3,5,6]$, we cannot conclusively state which of sets $C$ or $D$ are the correct ones. To avoid speculation, however, we can always compare the results from Ref. [3] to the experiment and discuss the MCD spectra in terms of SCFPPP method.

Such a comparison is made in Fig. 1 where the simulated MCD and absorption spectra are presented for the same values of $D_{0}$ and $A_{1} / D_{0}$ as those given in Table. In the calculations leading to curves in Fig. $1 \mathrm{~b}$ the small $B_{0}$ MCD contributions were neglected and the intensity distribution of an individual transition was approximated by a Gaussian. The corresponding experimental spectra without the background correction are those reported in Ref. [4].

A look at Fig. 1 reveals that the results of CNDO/S calculations [3] represented by broken lines are rather poorly related to MCD experiment and the observed differences are not due to simplifications used to simulate MCD spectra. Except for the region corresponding to $A_{\mathrm{g}} \rightarrow{ }^{1} T_{1 \mathrm{u}}$ transition, the discrepancies occur in the remaining high-energy part of MCD. They are apparently due to incorrect sign pattern of $A_{1} / D_{0}$ ratios. On the other hand, the results of SCFPPP method represented by solid lines in Fig. $1 \mathrm{~b}$ lead to reasonably good curve-to-curve agreement with experiment. We wish to note, however, that SCFPPP calculations leading to solid line spectra in Fig. 1b were done without spin-orbit couplings suggested in the original experimental paper [4] to play a role in a interpretation 


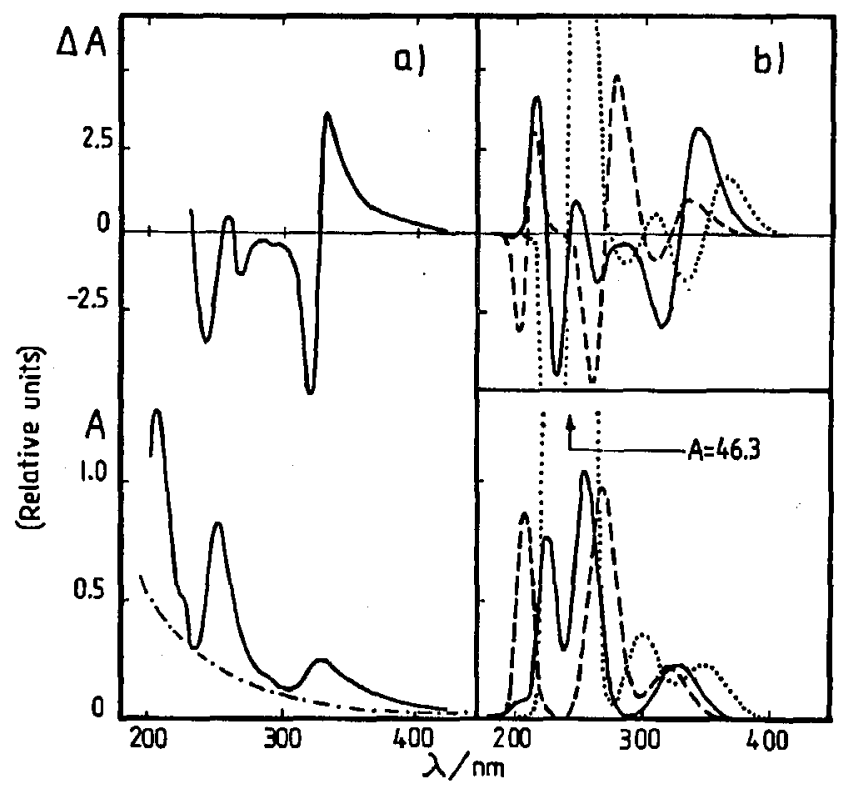

Fig. 1. The experimental (a) and the theoretical (b) MCD and absorption spectra: $(-$ SCFPPP with $\mathrm{CI}=900,(---) \mathrm{CNDO} / \mathrm{S}$ with $\mathrm{CI}=171$ and $(\cdot \cdots)$ with $\mathrm{CI}=196$.

of MCD data. Since the spin-orbit coupling effects are usually small, the minor redistribution of MCD would be expected when ${ }^{1} T_{1 u}$ and ${ }^{3} T_{1 u}$ electronic manifolds would be involved in the spin-orbit coupling.

Returning to Table we wish to note that SCFPPP method applied in the full CI treatment gives very small value of $D_{0}\left(2 T_{1 u}\right)$ as compared to other states of the same symmetry parentage. It should be also noted that the relations: $D_{0}\left(1 T_{1 \mathrm{u}}\right)>$ $D_{0}\left(2 T_{1 \mathrm{u}}\right)$ and $D_{0}\left(2 T_{1 \mathrm{u}}\right)<D_{0}\left(3 T_{1 \mathrm{u}}\right)$ are predicted by the QCFF/PI method used with 266 configurations [2]. Therefore, it is interesting to examine whether or not such a small intensity of $2 T_{1 \mathrm{u}}$ state results from the effect of size of CI basis used in our calculations. To do so, we calculated $A_{1} / D_{0}$ and the relative values of $D_{0}$ as functions of the number of configurations. The results of such calculations are given in Fig. 2. We included all configurations resulting from the single electron promotions from the (all) filled $\pi$ molecular orbitals to a given empty shell. The symmetry of the last accepting shell is indicated in the abscissa of Figs. $2 \mathrm{a}$ and $2 \mathrm{~b}$. The number on the right-hand side of each plot labels the electronic $T_{1 \mathrm{u}}$ states in order of increasing energy. In the calculations the semi-empirical parameters, i.e., the repulsion integrals $\left(\gamma_{i k}\right)$ and resonance integrals $\left(\beta_{i k}\right)$ were parametrized as $[1,7,8]$ :

$$
\begin{aligned}
& \gamma_{i k}=14.3994\left(1.294+R_{i k}\right)^{-1}, \\
& \beta_{i k}=-2018.0 \exp \left(-b R_{i k}\right),
\end{aligned}
$$



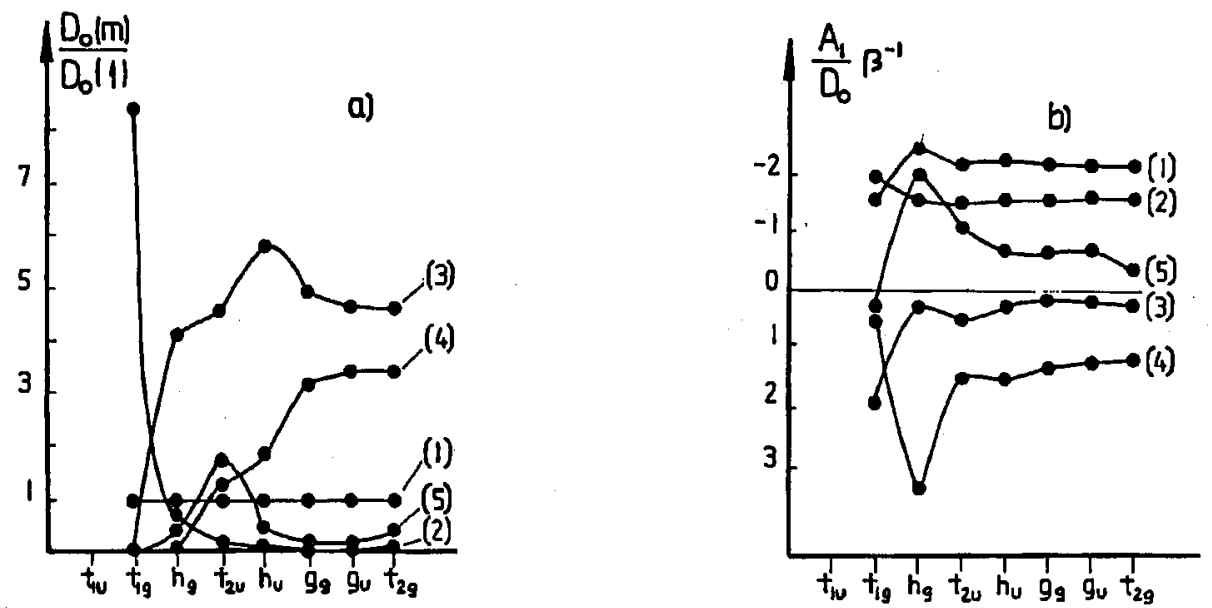

Fig. 2. The relative values of $D_{0}$ (a) and $A_{1} / D_{0}$ ratios (b) as a function of SCFPPP calculations.

$b$ equals $5.007 \AA^{-1} . R_{i k}$ is the distance between $i$-th and $k$-th carbon nuclei known from a structural modified neglect of differential overlap (MNDO) study [9] and applied here without modification.

Figure 2 shows that the absorption coefficients and the $A_{1} / D_{0}$ ratios undergo substantial changes as the number of configurations varies. Specifically, these changes cannot be ignored unless all the occupied $\pi$ molecular orbitals and the highest energy empty shell of $h_{\mathrm{u}}$-symmetry are involved in CI treatment. At this level, the calculated energies (not given) are less than 6 percent larger than those available from experiment and the calculations based on the full CI treatment. The subsequent increase in the number of configurations only slightly modifies both $A_{1} / D_{0}$ and $D_{0}$ quantities. Nevertheless, the full CI treatment should be favored, especially when the problem addresses the calculations of the Herzberg-Teller coupling parameters needed to interpret the vibronic structure of $A_{\mathrm{g}} \rightarrow T_{1 \mathrm{u}}$ transitions [4]. In such calculations the status of accuracy of the electronic function involved in the vibronic coupling plays an essential role [10]. Thus, our objections regarding MCD results from Ref. [3] would apply also to those reported most recently [5].

Looking again at Fig. 2a we see that $D_{0}$ for the $A_{1} \rightarrow 2 T_{1 \mathrm{u}}$ transition drops rapidly when the number of configuration increases. Therefore, the $2 T_{1 \mathrm{u}}$ state is expected to be weak and not observed in the absorption spectrum of $\mathrm{C}_{60}$ molecule under typical spectral resolution. However, $2 T_{1 \mathrm{u}}$ state has quite large positive magnetic moment. Therefore, its role in an interpretation of the vibronically induced MCD features [4] has to be anticipated. A similar conclusion can be drawn when the problem concerns the magnetic vibrational circular dichroism (MVCD) spectra of the $\nu_{4}=1430 \mathrm{~cm}^{-1}$ and $\nu_{3}=1183 \mathrm{~cm}^{-1}$ infrared active vibrations of $\mathrm{C}_{60}$ molecule [11]. Since the theoretical model dealing with MVCD of the triply degenerate vibrations already exists [12], the MVCD problem for $\mathrm{C}_{60}$ molecule will be studied in one of our subsequent papers. 


\section{Conclusion}

In this paper we have studied the effect of configuration interaction on the magneto-optical properties of $\mathrm{C}_{60}$ molecule in the low-energy $T_{1 \mathrm{u}}$-symmetry states. For purposes of this study we have used the SCFPPP method suitably adapted to deal with the molecules which have the three-dimensional $\pi$-systems. Within this approach we have shown that the SCFPPP method applied in the CI treatment comprising all single excited configurations accounts correctly for the main features of the MCD and absorption spectra reported to date for the $\mathrm{C}_{60}$ molecule.

\section{Acknowledgment}

We would like to thank the Committee for Scientific Research (project 2.P303.106.04) for financial support of this work.

\section{References}

[1] M. Pilch, M. Pawlikowski, Chem. Phys. 172, 277 (1993).

[2] F. Negri, G. Orlandi, F. Zerbetto, Chem. Phys. Lett. 144, 31 (1988).

[3] G. Marconi, P.R. Salvi, in: Fullerene: Status and Perspectives, Ed. C. Taliani, Vol. 2, World Scientific 1992, p. 99.

[4] Z. Gasyna, P.N. Schatz, J.P. Hare, T.J. Dennis, H.W. Kroato, R. Taylor, D.R. Walton, Chem. Phys. Lett. 183, 283 (1991).

[5] G. Marconi, P.R. Salvi, Chem. Phys. Lett. 202, 335 (1993).

[6] F. Negri, G. Orlandi, F. Zerbetto, J. Chem. Phys. 97, 6496 (1992).

[7] V.A. Kupryevich, Int. J. Quant. Chem. 1, 561 (1967).

[8] A. Golębiewski, Chemia kwantowa zwiqzków organicznych, PWN, Warszawa 1973.

[9] M. Froimowiz, J. Comp. Chem. 9, 1129 (1991).

[10] J. Del Bene, H.H. Jaffe, J. Chem. Phys. 48, 1807 (1968).

[11] Cheok N. Tam, B. Wang, T.A. Keiderling, Chem. Phys. Lett. 198, 123 (1992).

[12] M. Pawlikowski, Chem. Phys. 127, 121 (1988). 